\title{
Blockchain and AI-based Solutions to Combat Coronavirus (COVID-19)-like Epidemics: A Survey
}

\author{
Dinh C. Nguyen, Ming Ding, Pubudu N. Pathirana, Aruna Seneviratne
}

\begin{abstract}
The beginning of 2020 has seen the emergence of coronavirus outbreak caused by a novel virus called SARS-CoV2. The sudden explosion and uncontrolled worldwide spread of COVID-19 show the limitations of existing healthcare systems to timely handle public health emergencies. In such contexts, innovative technologies such as blockchain and Artificial Intelligence (AI) have emerged as promising solutions for fighting coronavirus epidemic. On the one hand, blockchain can combat pandemics by enabling early detection of outbreaks, protecting user privacy, and ensuring reliable medical supply chain during the outbreak tracking. On the other hand, AI provides intelligent solutions for identifying symptoms caused by coronavirus for treatments and supporting drug manufacturing. Motivated by these, in this paper we present an extensive survey on the use of blockchain and AI for combating coronavirus (COVID-19) epidemics based on the rapidly emerging literature. First, we introduce a new conceptual architecture which integrates blockchain and AI specific for COVID-19 fighting. Particularly, we highlight the key solutions that blockchain and AI can provide to combat the COVID-19 outbreak. Then, we survey the latest research efforts on the use of blockchain and AI for COVID-19 fighting in a wide range of applications. The newly emerging projects and use cases enabled by these technologies to deal with coronavirus pandemic are also presented. Finally, we point out challenges and future directions that motivate more research efforts to deal with future coronavirus-like epidemics.
\end{abstract}

Index Terms-Blockchain, Artificial Intelligence (AI), security, privacy, machine learning, deep learning, coronavirus (COVID19), SARS-CoV-2, epidemic.

\section{INTRODUCTION}

The coronavirus (COVID-19) outbreak in late 2019 comprises a serious threat around the world [1]. The severity of the epidemic was so huge that the World Health Organization (WHO) was compelled to declare it as a pandemic within a month of its wide-scale expansion. The virus spread causes the global economic shock with the massive interruptions of many sectors such as supply chain, industry, insurance, agriculture, transport, and tourism, forcing governments and owners to shut stop operations on a worldwide scale [2]. According to the Organisation for Economic Cooperation and Development (OECD), the global economy could grow at its slowest rate

*This work was supported in part by the CSIRO Data61, Australia.

Dinh C. Nguyen is with School of Engineering, Deakin University, Waurn Ponds, VIC 3216, Australia, and also with the Data61, CSIRO, Docklands, Melbourne, Australia (e-mail: cdnguyen@deakin.edu.au).

Ming Ding is with Data61, CSIRO, Australia (email: ming.ding@data61.csiro.au).

Pubudu N. Pathirana is with School of Engineering, Deakin University, Waurn Ponds, VIC 3216, Australia (email: pubudu.pathirana@deakin.edu.au).

Aruna Seneviratne is with School of Electrical Engineering and Telecommunications, University of New South Wales (UNSW), NSW, Australia (email: a.seneviratne@unsw.edu.au).

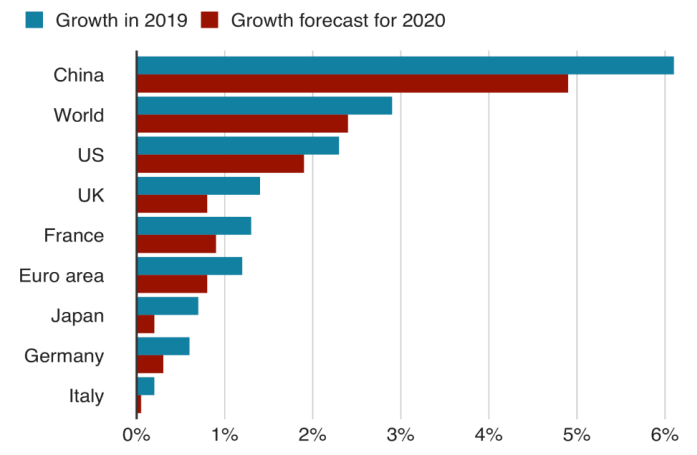

Fig. 1: The impact of COVID-19 on global economic growth (Source: OECD).

since 2009 in this year [3] due to the coronavirus outbreak as the forecasts in Fig. 1. As the number of infections rises, many governments around the world have instituted drastic lock-downs and curfews and called for social distancing and work from home to reduce the rate at which the virus spreads.

With the deadly coronavirus spreading globally, every attempt is being made to ensure help for victims as well as to stop the spread. As governments scramble to address these problems, technology-empowered solutions can help deal with the worldwide health crisis. Applications of innovative technologies such as Blockchain and Artificial Intelligence (AI) could have the answers in response to coronavirus crisis [4], [5], [6]. While blockchain can combat pandemics by enabling early detection of outbreaks, fast-tracking drug delivery, and protecting user privacy during the treatment, AI provides intelligent solutions for identifying symptoms caused by coronavirus for treatments and supporting drug manufacturing. It is also argued that the present coronavirus crisis should be seen as a "call to arms for the technology industry" where blockchain and AI may be the key enablers for radically changing the landscape of crisis response and the management of the coronavirus outbreak [7], [8].

\section{A. Motivations of using blockchain and AI for coronavirus fighting}

1) Limitations of current healthcare systems: The COVID19 outbreak could push the existing healthcare systems to their limits. At present, there is a lack of a trusted data surveillance system that would instantly give relevant healthcare organizations the information they need about potential outbreaks. In fact, most of the current coronavirus information comes from separate sources such as the public, hospitals, clinical labs with a large amount of inaccurate data without 
being monitored thoroughly. The use of untrusted information makes it challenging for potential outbreak identification and quarantine. Another limitation is the current time-consuming and in-accuracy coronavirus detection procedure that often takes some days to complete the virus tests. It is unacceptable with the rapid spread of coronavirus. How to achieve a fast-checking coronavirus detection with high accuracy is an urgent need. Furthermore, it is very challenging to process coronavirus data with complex patterns and large volumes by using human-depending medicine tools. Finally, the mass surveillance on the public to track coronavirus raises critical privacy issues. For example, the Israel government recently allows healthcare organizations to track citizens' phones without requiring a court order in an effort to curb the spread of the COVID-19 coronavirus [9]. However, this measure has faced criticism from human rights and privacy experts because it can reveal sensitive personal information of citizens, prompting major concerns of privacy and civil liberty violations. Therefore, how to develop solutions that enable to instantly monitor coronavirus spread while protecting user privacy is highly necessary to solve this emergency epidemic.

2) Benefits of using blockchain and AI for solving the coronavirus epidemic: Blockchain and AI are able to provide viable solutions to cope with the coronavirus epidemic from various aspects. Blockchain that has proved its success in many real-world applications can be applied to support coronavirus outbreak fighting. A possible application is an outbreak tracking such that blockchain can be leveraged globally to track the spread of the coronavirus infections via a blockchain network deployed on mobile devices of citizens. An important feature of blockchain is the ability to preserve user privacy which can coronavirus spread tracking, enabling early detection of epidemics while avoiding user information exposure. Moreover, blockchain can actively simplify the process of fast-tracking drug trials, and recording and tracking all fundraising activities and donations, in a transparent and trusted fashion, which can support the management of outbreaks and treatment.

On the other hand, AI can help tackle the coronavirus epidemic in several ways. AI can be used to detect virus and predict how the virus is going to spread by analysing the combined information of environmental conditions, access to healthcare, and the way it is transmitted. Based on that, AI can identify coronavirus within localized outbreaks of the disease and help reveal the nature of the virus [10]. The coronavirus can cause severe symptoms such as pneumonia, severe acute respiratory syndrome, and kidney failure. AI-based algorithms such as genome-based neural networks already built for personalized treatment can prove very useful in controlling these adverse events or symptoms caused by a coronavirus, especially when the impact of the virus depends on immunity and the genome structure of individuals and no standard treatment can treat all symptoms effectively at present. Further, the use of AI may be very helpful in identifying the relationship of novel coronavirus and related viruses such as SARS [11] to accelerate the finding of a new vaccine. Finally, AI approaches can automatically build a model or relationship between treatments documented in healthcare records and the eventual patient outcomes. These models can quickly identify diagnosis and treatment choices that assist to guide the process of developing clinical guidelines for future coronavirus-like epidemics. With these promising benefits, recently the White House urgently calls for using AI to aid the US Government's response to the coronavirus pandemic [12].

\section{B. Contributions}

It is believed that blockchain and AI could potentially have a considerable impact on the management of pandemic situations and the dissemination of treatment [13]. To date, the literature lacks comprehensive reviews and studies on the applications of blockchain and AI for coronavirus (COVID-19) fighting. The purpose of this paper is to provide the readers with an initial picture and road map of how blockchain and AI can support to solve the coronavirus epidemic and related healthcare crisis. Therefore, in this paper we present an extensive survey on the applications and use cases of blockchain and AI technologies specific to coronavirus (COVID-19) pandemic by using the rapidly emerging literature and the latest research reports. To this end, our survey provides the following contributions:

1) We introduce a conceptual systematic architecture that integrates blockchain and AI for coronavirus (COVID-19) fighting, aiming to provide the key solutions in response to coronavirus epidemic.

2) We identify a number of specific applications using these technologies for solving coronavirus-related issues detection. Particularly, we highlight the potentials of blockchain and AI through a comprehensive analysis and discussion in different applied scenarios.

3) We explore the latest use cases and projects using blockchain and AI for coronavirus fighting.

4) Based on the extensive survey, we identify possible research challenges and future directions to encourage scientists and stakeholders to put more efforts in developing innovative solutions to combat the future coronaviruslike epidemics.

\section{Organization}

The structure of this survey is organized as follows. Section II presents the background of coronavirus epidemic, and two key technologies used to combat the crisis, including blockchain and AI. We also introduce a conceptual architecture that integrates blockchain and AI for coronavirus fighting. In Section III, we analyse the role of blockchain for coronavirus fighting via five key solutions, including outbreak tracking, user privacy protection, safe day-to-day operations, medical supply chain, and donation tracking. Next, in Section IV, we present a review on the use of AI for coronavirus epidemic fighting. AI can support by providing five main applications, namely outbreak estimation, coronavirus detection, coronavirus analytics, vaccine/drug development, and prediction of future coronavirus-like outbreak. Section V summarizes the most popular use cases on blockchain and AI adaption in response to coronavirus fighting. Then, we point out some important challenges and potential future directions for applying these technologies in the context of healthcare sector for 
epidemic, in Section VI. Finally, Section VII concludes the paper.

\section{BACKGROUND}

In this section, we provide briefly the coronavirus pandemic, and then summarize blockchain and AI technologies used for fighting coronavirus. Then we introduce a systematic architecture for coronavirus fighting.

\section{A. Coronavirus (COVID-19) pandemic}

The first outbreak of the COVID-19 virus epidemic took place in Wuhan, a city of 11 million, the capital of Hubei Province, China, starting in December 2019 [14]. It is not yet clear which animal the new coronavirus is associated with, although some data suggests it is similar to those found in bats. Coronaviruses affect the breathing passages, or respiratory tract. The number of infected citizens grew exponentially until Chinese authorities declared a complete lockdown of the affected region, the 60-million-people Hubei province. Symptoms can be mild like the common cold, including a runny nose and cough or they can be more severe and can cause difficulty in breathing. Other common symptoms includes fever, cough, and feeling tired. Some people become seriously ill but this seems more likely in the elderly and those with health problems [15]. At present, the new coronavirus has infected more than 110,000 people in at least 110 countries and territories globally [16]. The COVID-19 disease is spreading rapidly around the world, with countries like Italy, Iran and South Korea reporting more than 100,000 cases up to now. Other European countries like France, Germany and Spain have also seen a recent spike beyond 5,000 cases [17]. The COVID-19 epidemic results in great harm to people's daily life and country's economic development. Governments have locked down cities, restricted movements of millions and suspended business operations that will slow down the global economy in the coming years. Undoubtedly, the virus outbreak has become one of the biggest threats to the global economy and financial markets [18], [19].

\section{B. Blockchain}

Blockchain is mostly known as the technological platform behind Bitcoin [20]. The key concept of a blockchain is decentralization. In fact, its database does not place in a central location, but is distributed across a network of participants (i.e. computers). This decentralized concept provides high robustness and security for database stored on blockchain with no single-point failure. Importantly, blockchain is visible to each member in the network. This is enabled by a mechanism called consensus which is a set of rules to ensure the agreement among all participants on the status of the blockchain ledger. A concept of how blockchain works is shown in Fig. 2. In general, blockchains can be classified as either a public (permission-less) or a private (permissioned) blockchain [21]. A public blockchain is accessible for everyone and anyone can join and make transactions as well as participate in the consensus process. The best-known public blockchain applications include Bitcoin and Ethereum [22]. Private blockchains

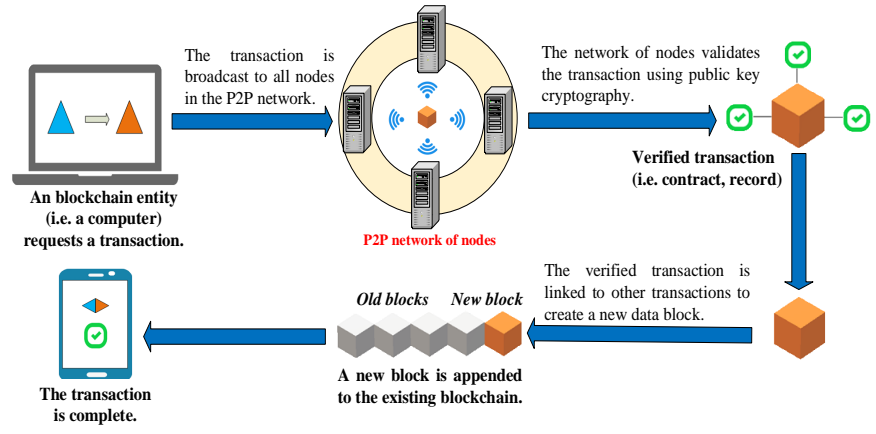

Fig. 2: The concept of blockchain operation.

on the other hand are an invitation-only network managed by a central entity. A participant has to be permissioned using a validation mechanism. Each blockchain consists of three main components, including data block, distributed ledger (database), and consensus algorithms.

Here, data block can be defined as a series of blocks, starting with a genesis block and interconnecting with every newly updated block to create a chain. Each block is strongly linked to the previous one via a hash label, which provides a robust interconnection among block and prevents any modification risks [23]. Meanwhile, the distributed ledger is a kind of database that is replicated over all network participants. It records and stores transactions which are created by users and ensured consensus among utilities by the mining process (i.e. Proof of Work-PoW). In the distributed ledger, each record contains a unique cryptographic signature decoupled with a timestamp which makes the ledger resistant to be modified. In terms of consensus algorithms, the process of transacting the block over the chain should not be controlled by any entity so that each block is managed by all participants with equal rights to avoid security issues, i.e. double-spending attacks. This can be done by a mechanism called consensus. From the view of blockchain, the consensus process mainly provides a guarantee for agreement on each data blockchain among entities. For instance, Bitcoin uses PoW algorithm [23] as a main consensus scheme for its transaction management. The nodes with high computational capability can join the mining process and compete with each other to be a first one to verify the block. In return, the winner can receive a certain amount of coin as the reward for its mining effort. Along with the advance of blockchain, there are some new consensus algorithms such as Proof-of-stake (PoS), Byzantine Faulty Tolerant (BFT) [24]. Blockchain has proved its success in healthcare and biomedical applications with promising performances in terms of healthcare data privacy [25], secure data management [26], [27], and transparent medical data storage [28], [29]. Therefore, it is possible to apply in solving healthcare issues related to coronavirus epidemic.

\section{Artificial Intelligence (AI)}

AI techniques have been used recently as a powerful tool for coronavirus data analytics, prediction and drug/vaccine discovery [30]. Recent studies show that AI has been mostly used for solving coronavirus-related issues via two key approaches: 


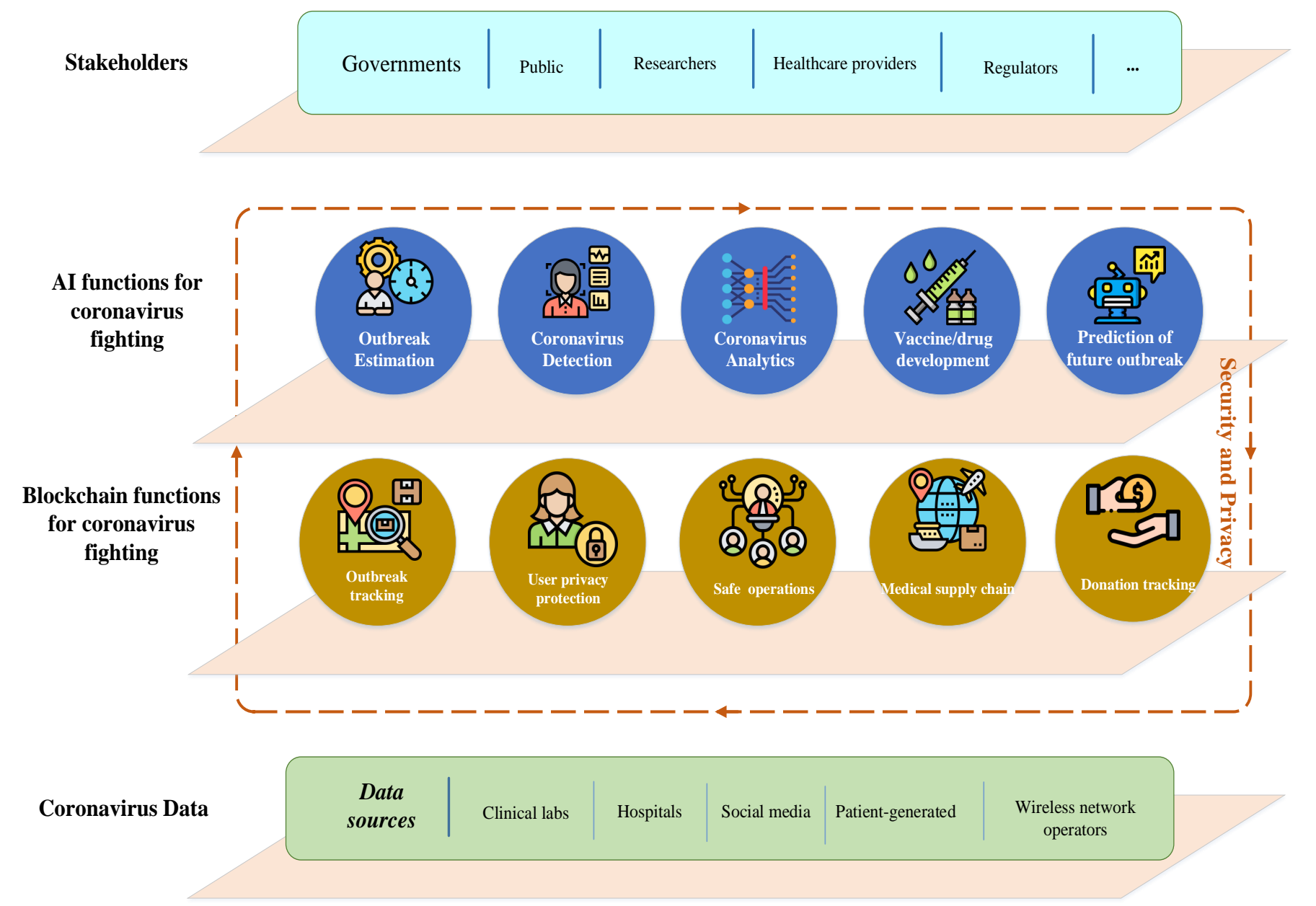

Fig. 3: Blockchain and AI for coronavirus fighting.

machine learning (ML) and deep learning (DL). ML is a subfield of AI. The objective of ML in general is to understand the structure of data and match that data into models that can be expressed and utilized by people [31]. ML algorithms instead allow computers to perform training on data inputs and employ statistical analysis solutions to output values that fall within a specific range. Based on this, ML can provide building models from sample data to automate decision-making processes using data inputs. In the coronavirus fighting, ML can be used for some services, such as facial recognition to detect infected people or on temperature detection on the human body for possible virus infection [32], [33]. For example, recently an AI company in the US specialising has utilized ML-powered interactive graphs are tracking the virus migration across China [34], for creating an alert system, whereby users will be able to receive information about whether an infected individual has travelled within their vicinity. This solution will help find infected individuals and provide medical resources to them.

Meanwhile, DL methodologies have been also exploited to implement intelligent coronavirus fighting solutions. Conceptually, DL uses multiple neural network layers in a deep architecture [35]. The neural network use multiple neurons connected via weighted connections among neural layers. For example, a basic deep neural network contains an input layer for receiving data samples, a single hidden layer for training and an output layer for generating training outcomes. Here, the number of hidden layers reflects the depth of the deep learning architecture. To generate the desired output, supervised or unsupervised learning techniques are used with the labeled or unlabeled data samples, associated with the adjustment of weight values among perceptrons [36]. DL is known for AI applications such as speech recognition, computer vision, image processing, object detection for healthcare applications [37], [38], [39] and thus coronavirus tackling can be a specific domain that DL can be applied for coronavirus fighting. Many AI companies have developed DL-enabled solutions to predict coronavirus infection and produce drugs and vaccines necessary for coronavirus disease [40].

\section{Proposed blockchain-AI architecture for coronavirus fight- ing}

In this paper, we present an architecture as shown in Fig. 3 that integrates blockchain and AI for coronavirus fighting. The architecture is conceptually organized into four layers, including coronavirus data sources, blocchain functions, AI functions, and stakeholders. The work flow is explained as follows.

Initially, all the data from clinical labs, hospitals, social media, and many other sources are consolidated and create raw data that subsequently develops in scale to big data. This 
data needs to be ensured privacy and security during the coronavirus outbreak tracking and analytics, by using blockchain. Here, blockchain can offer a number of viable solutions for coronavirus-related services such as outbreak tracking, user privacy protection, safe day-to-day operations, medical supply chain, and donation tracking. Secure data collected from the blockchain network is analysed using intelligent AI-based solutions. By using reliable prediction and accurate analysis ability on big data collected from coronavirus sources, AI can provide support for coronavirus fighting via five main applications, namely outbreak estimation, coronavirus detection, coronavirus analytics, vaccine/drug development, and prediction of any future coronavirus-like outbreak. Finally, at the top of the hierarchy comes the stakeholder layer which includes parties such as governments, healthcare providers who are benefiting from blockchain-AI solutions. Note that blockchain can build secure communication networks and protocols to establish a privacy-preserving, fast and reliable data exchange with the stakeholders thanks to its decentralized nature [41]. In the following sections, we present an extensive survey on the latest research efforts on using blockchain and AI for coronavirus epidemic.

\section{BLOCKCHAIN-BASED SOLUTIONS FOR CORONAVIRUS} FIGHTING

In this section, we analyse the role of blockchain for coronavirus fighting via five key solutions, including outbreak tracking, user privacy protection, safe day-to-day operations, medical supply chain, and donation tracking.

\section{A. Outbreak tracking}

Blockchain can provide feasible solutions for tracking the coronavirus outbreak. Indeed, the blockchain can realize provide data visualization tools for deadly coronavirus monitoring. Blockchain can be seen as distributed database ledgers that can receive multiple updates in near real-time and store them on blocks linked together in a trusted and immutable manner. In the coronavirus epidemic, blockchain is potential to support the thousands of coronavirus victims by recording immutably patient symptoms of infection [42]. This is highly important to track the spread of coronavirus because some patients can deliberately declare wrong their symptoms for avoiding hospital visits, which can lead to patient quarantine failure. Governments and healthcare organization can monitor potential patients at every stage via blockchain with high reliability and accuracy. It can also track patient moments, provide real-time data about affected areas and direct fighting efforts [43]. Besides, blockchain is also useful to implement tracking of person movement in virus-free zones [44]. Information of safe zones such as population, location, current coronavirus outbreak status is recorded via a chain of blocks, each block can store an update of the outbreak in a certain time. Note that such data can be collected from surveillance providers who use a combination of technologies like AI and geographical information systems (GIS). Based on that, healthcare service professionals can identify the free-virus zones from the infected ones to implement quarantine requirements [45].
Blockchain hence can provide practical approaches to protect the community against virus spread.

Another major problem in the coronavirus pandemic at this time is the outbreak of fake news circulated through social media channels and websites. We are facing an unprecedented crisis of public understanding. Last month, Turkey and North Korea have been under scrutiny for claiming they have no diagnosed cases, but in fact the actual statistic from the WHO is different [46], while the accuracy on the case number given by Iran has been called into question, with lower numbers of infected cases and deaths. Nowadays, people rely on social media platforms such as Facebook, Twitter to update the coronavirus pandemic, but these platforms are facilitators and multipliers of COVID-19-related misinformation. The incorrect information transmission really increases public confusion about who and what information sources to be trusted. The outbreak of fake news also generates fear and panic due to unverified rumours and exaggerated claims, and promotes racist forms of digital vigilantism and scapegoating [47]. Governments, digital corporations and public health authorities should find solutions to verify and authenticate any information and news related to the pandemic which plays an important role in controlling public panic and facilitating coronavirus management. Blockchain can provide the potential to ensure the scrutiny of data accuracy by its ability to validate information, immutable data storage, real-time data updates. More importantly, blockchain eliminates the need of third parties thanks to its decentralization [48], which can significantly reduce the risk of information modification and fake news and then increase the information reliability to the public and healthcare professionals. Blockchain can be a platform for coronavirus tracking, ensuring that the information processed through its blockchain network is accurate, transparent, tamper-free, and trustworthy. As a result, governments can update better on coronavirus epidemic for better management policies, such as disease estimation, quarantine of potential areas, and tracking the virus spread. In summary, blockchain can support outbreak tracking by the following solutions:

- Enforcing a standard of common data reporting

- Establishing a single source of truth

- Monitoring effectively quarantine cases, at home or at medical facilities

- Enabling location tracking of the patients via real-time routine checking and recording on blocks, without impacting data privacy

\section{B. User privacy protection}

In an attempt to curb the spread of the virus, many solutions have been considered around the world. For example, in South Korea, the government agencies have exploited personal data for coronavirus fighting, including using surveillance-camera footage, credit card purchase history and smartphone location data [49]. Even, the government also posted detailed location histories of each coronavirus patient including where they stayed before being tested positive. On the one hand, such strategies can be helpful in tracing the movements of the confirmed coronavirus victims to monitor transmission chains. 
However, this also reveal sensitive personal information to the public, raising serious data leakage concern, which can discourage citizens from collaborating with healthcare organizations for virus testing. In the emergency situations like coronavirus pandemics, user privacy must be weighed against other considerations, like saving lives. How to balance the value of upholding public interest in fighting mass virus infections and the value of protecting individual privacy and human rights is of paramount importance for any coronavirus healthcare policies in this epidemic. Blockchain can come as a promising solution to address privacy protection issues during the coronavirus fighting. This ability is enabled by accountability and transparency that blockchain can provide on the data access. In fact, blockchain is able to trace the data operation to detect any malicious data usage or illegal access behaviours without permission. In this way, each blockchain node (i.e. a hospital or a government agency) can keep track the data flow on the blockchain in a trusted and transparent manner. The efficiency of this concept has been also investigated in preserving personal information and private data for healthcare applications [50], [51].

In the coronavirus pandemic, blockchain can be used to record patient information with symptoms, locations, historic health conditions with high privacy. This can be achieved by the trust and decentralization features of blockchain. Each healthcare record is always time-stamped with signature for data proof and encrypted before storing on the block, which can prevent modifications and changes from malicious users and adversarial attacks. This would protect the data privacy for all users, including the infected ones. Furthermore, the data block is decentralized over the distributed network of governments, healthcare professionals, and users [52]. That means the data is controlled and monitored by all parties, and any data retrieval and data updates are automatically reflected on the network for data flow tracking.

Blockchain is also able to provide privacy for surveillance networks for coronavirus tracking [53]. In some Asian countries where ubiquitous surveillance systems are deployed in the community to trace the virus spread for addressing the coronavirus crisis. In such contexts, the user privacy can be ensured by using distributed blockchain that can be run over the user nodes (i.e. mobile devices) to collect data but keep personal information secret. This would provide adequate transparency, accountability and reliability on data for everyone.

\section{Safe day-to-day operations}

In the coronavirus crisis, blockchain has emerged as a promising platform that enables to conduct day-to-day activities in virtual environments to reduce the risk of virus contraction. Here we consider two popular daily operations with user-to-user and cross-border operations.

1) User-to-user operations: In light of the ongoing coronavirus pandemic, customer services via virtual environments such as digital blockchain networks would become feasible to mitigate the spread of virus. For example, the UAE's Ministry of Community Development has switched to using digital channels via blockchain for civil services [54].
Instead of visiting government offices and service centres for paper works, the government services such as the digital authentication of official certificates and other documents are implemented by blockchain. The trial results indicate that blockchain can process 2,919 different types of documents, which is even faster than the traditional working approaches. More importantly, this blockchain-based solution really helps to reduce the risk of infection from face-to-face contact that accordingly mitigates the possibility of virus spread in the community.

Furthermore, economic activities can be continued using virtual platforms based on digital blockchain. In fact, electronic payment among customers and companies now can be performed via blockchain instead of cash which may be a source of coronavirus spread [55]. Blockchain is well known to make digital money such as Bitcoin [56] which proves efficiency in certain digital transactions such as digital auction. In the coronavirus crisis that people should not use banknotes as Merchants are encouraging, electronic payment via blockchain may be an ideal choice. The decentralized concept of blockchain ensures payments in a secure, transparent, and immutable interface, which is free from human interaction. Another example is Ant Financial which has been launching a blockchain-enabled online bid opening system to replace the cash-based approaches [57]. It allows customers to perform in contactless bidding from remote locations when they take quarantine measures to combat the COVID-19 outbreak. This bidding system is operated on a consortium blockchain platform which ensures that materials and processes of bid openings are tamper-proof, and the contactless bidding is transparent and trustworthy.

2) Cross-border operations: With the negative impact of coronavirus pandemic, the international business operations can be interrupted due to the lack of trust in collaborative business, verification inefficiency, the lack of information sharing and difficulty of timely supervision among countries [58]. A cross-border blockchain network can play a crucial role in creating a virtual environment for interconnecting countries and regions with high trust and efficiency in economic activities and healthcare supports [59]. Further, thanks to its decentralization feature where blockchain nodes can be held by companies and public authorities, the stable process of good exchange and health supports is also ensured. Another challenge for governments during large-scale coronavirus disasters is the interrupted distribution of cross-border payments. Blockchain can help to overcome this issue. For example, the United Nations World Food Program relied on blockchain for money transfer to more than 10,000 Syrian refugees [60], auditing online beneficiary spending, which reduces bank transfer fees by nearly $98 \%$. This would save millions of dollars for aid projects, like coronavirus-fighting programmes. From a high-level discussion based on the above examples, the use of blockchain for cross-border operations can be implemented specifically in the coronavirus crisis as the following key steps:

- Governments and international health organizations should implement a virtual blockchain-based platform that will collaborate local hospitals, medical centres, and 
health organizations in potential zones into which the virus may spread, without worrying about the risk of virus contraction.

- Local hospitals and clinical facilities will be able to record medical data about patients who coronaviruslike symptoms with specific patient identification on blockchain.

- Medical data will be further traced by the healthcare organizations to predict the spread of the virus using collected medical statistics.

- Based on that, countries will be able to strengthen their preventive measures in the areas where the virus can potentially spread, i.e. preparing more medical staff, providing more medical supplies.

\section{Medical supply chain}

Blockchain has proved extremely useful in supply chain applications such as goods supply chain, trading supply chain [61], [62], [63], [64]. In this pandemic crisis, maintaining a continuous supply of medicines and food has become a challenge for the healthcare sector. The blockchain technology can help the supply chain companies in achieving a fast flow of supply by tracking the flow from the origins to the destinations in a trusted and reliable manner.

Recently, Alipay, along with the Zhejiang Provincial Health Commission and the Economy and Information Technology Department, China has launched a blockchain-based platform that allows users to track the demand and the supply chains of medical supplies [65]. This consists of the recording and tracking of coronavirus epidemic fighting materials, such as masks, gloves and other protective gear. The company claims that blockchain can ensure high trust for the medical supply chain by secure linking among blocks and transactions, supply chain data privacy due to blockchain encryption, and fast data flow thanks to blockchain decentralization. When an outbreak occurs, a quick reaction and fast supply chain is the most important weapons authorities have to tackle the problem. By using blockchain, the supply chain issues can be solved, helping to save thousands of lives and billions of dollars [66]. In summary, blockchain can offer five key solutions to support the medical supply chain in the coronavirus crisis:

- Product requirements: provide a solution to update realtime demands and medical factories for fast response (i.e. supply chain rate adjustment).

- Supply creditability: provide a solution to control the quality of goods from the factory side, i.e. product specification, supply volumes.

- Transportation tracking: Goods, medical supplies need to be traced to guarantee transparency in the medical supply chain, which can be done by a blockchain network with transaction recording and monitoring capabilities.

- Financial payments: blockchain can be used as a payment platform between the suppliers and the customers (i.e. users in quarantine areas). All digital payments are recorded on the blockchain with timestamp, signature without being changed or modified.

- Customs certifications: customer behaviours such as buying, payment actions can be reflected on the blockchain with digitally signed certificates that shows the negotiation between the suppliers and the users.

\section{E. Donation tracking}

The potential of blockchain in donation tracking applications has been investigated in recent works [67], [68]. In the coronavirus crisis, donation is one of the most important activities to support livings and healthcare services for infected victims. A critical question raised is how to track the donation activities to ensure that donated goods, money are transferred to the targeted victims. Blockchain can be a feasible solution [69]. Indeed, the donation process can be traced through blockchain which issues signature and certificate to mark each of the donation updates, i.e. when, where, donation volumes, list of targeted receivers, etc. As an example, blockchain has been recently applied to trace the donation of protective gear like N95 masks to support citizens in China [70]. Blockchain also allows all parties such as suppliers, healthcare professionals, charity organizations to monitor the progress of donations. Any updates will be informed to all related parties to ensure transparency and trust over the donation network. The Italian Red Cross is now also seeking Bitcoin donations to purchase highly needed medical equipment to infected areas [71]. Binance Charity, a blockchain-based donation platform [72], also launches a million-dollars-campaign to seek donations via blockchain coins that are then exchanged to purchase supplies for supporting affected countries and regions.

\section{AI-BASED SOLUTIONS FOR CORONAVIRUS FIGHTING}

In this section, we present a review on the use of AI for coronavirus epidemic fighting. AI can support by providing five main applications, namely outbreak estimation, coronavirus detection, coronavirus analytics, vaccine/drug development, and prediction of future coronavirus-like outbreak.

\section{A. Estimation of coronavirus outbreak size}

AI can help combat COVID-19 spread by its ability to estimate the coronavirus outbreak size through analysing people's phone usage patterns. In fact, when people are sick, dead due to coronavirus infection or taking care of family members, they tend to change their patterns of phone usage, which can be detected by AI algorithms run on the datasets provided by wireless operators [73]. For example, if we can get access to the wireless operators data in Wuhan from November 2018 to March 2020, then we may be able to find out the activity patterns of cell phones. These patterns can include abnormal usage (i.e. frequent calls in early morning) from sick people, non-calling activities from people died from coronavirus infection, or new phone appearance in another city from people sneaked out of the lockdown city. In these situations, AI can analyse such different activities which can reveal the patterns of mobile users accordingly [74]. For instance, ML is able to model and predict the personalized diverse activities of a user through learning from his phone usage records [75]. Furthermore, DL would be a promising AI technique that relies on deep data learning and highperformance model prediction, aiming to estimate accurately 
the mobile application usage, i.e. abnormal calling behaviours, phone service inactivity [76]. Therefore, such AI applications can be applied in the context of coronavirus epidemic to reveal the user patterns so that the agencies can estimate the size of coronavirus outbreak.

Moreover, a mobile operator can detect and track infected people's movement to predict the outbreak using AI analytics based on their cellular network location data. For example, an American mobile company develops a tracking system that can collect mobile location data of users in up to 500 U.S. cities with user privacy awareness [77]. AI can analyse the user movement patterns to estimate the locations where crowds are still gathering and specify certain geographic quarantine areas. Meanwhile, stemming from the fact that people in some Chinese cities rely on their mobile phone for daily public transportation, Chinese railway authorities use AI to track the customers movement and identify people who were near a passenger infected with COVID-19 [73]. Human mobility patterns can be also estimated by using AI analytics based on geo-located Twitter social media data records [78]. This solution can trace the geographical location of infected patients and keep track their movement to predict the coronavirus spread.

\section{B. Coronavirus Detection}

Some recently emerging solutions using AI for coronavirus detection have been proposed in the literature. A measure is to detect temperature on human face so that we can identify potential symptoms of COVID-19 disease. AI can come up with solutions thanks to its capability in face recognition applications. The work in [79] presents an improvement in masked face detection using AI with real-world datasets in response to the coronavirus epidemic. Through deep learning, the dataset is trained to build an accurate masked face detection model, which then serves for the masked face recognition task, aiming to determine whether a person is wearing a mask. Different from this study, the authors in [80] rely on breathing characteristics to detect potential people infected with COVID19. In the coronavirus detection, accurate identification of the unexpected abnormal respiratory pattern of people in a remote and unobtrusive manner is highly significant. Deep learning (DL) has been used for respiratory pattern classification, aiming to to classify six clinically significant respiratory patterns related to COVID-19 (Eupnea, Tachypnea, Bradypnea, Biots, Cheyne-Stokes and Central-Apnea).

Another methodology is the thoracic CT image analysis to detect Coronavirus positive patients [81]. Non-contrast thoracic CT has been shown to be an effective tool in detection, quantification and follow-up of corona-similar diseases. Given a potentially large number of thoracic CT exams, a detection system is proposed that uses both robust $2 \mathrm{D}$ and $3 \mathrm{D} \mathrm{DL}$ models coupled with existing AI models and combining them with clinical understanding. The proposed model is able to detect suspected COVID-19 thoracic CT features with high accuracy. CT image analysis is also implemented in [82] to early screen COVID-19 patients. The finding results indicate that the manifestations of computed tomography (CT) imaging of COVID-19 had their own characteristics, which are different from other types of viral pneumonia, such as Influenza-A viral pneumonia. Using these features, a 3D DL-based a locationattention classification model is developed using a pulmonary CT image set for symptom detection, showing a high accuracy with $86.7 \%$ for three groups: COVID-19, Influenza-A viral pneumonia and healthy cases. The work in [83] also uses image analysis-based techniques for coronavirus detection. An array of convolutional neural network based models (ResNet50, InceptionV3 and InceptionResNetV2) is leveraged to analyze the chest X-ray radiographs, aiming for detecting coronavirus pneumonia infected patients. The ResNet50 model can provide the highest classification performance with $98 \%$ accuracy among the other two proposed models, as confirmed from simulations.

To further improve the accuracy of COVID-19 detection, the authors in [84] leverage a deep convolutional neural network that is able to automatically extract features starting from the genome sequence of the virus. Experiments using the 2019nCoVR dataset show a correct classification performance in detecting SARS-CoV-2, and distinguishing it from other coronavirus strains, such as MERS-CoV, SARS-CoV. Meanwhile, a research effort in [85] is toward diagnostic uncertainty verification in the COVID-19 detection. A Bayesian Convolutional Neural Network (BCNN) is considered to estimate uncertainty in DL solutions. The objective of this study is to improve the diagnostic performance of the human-machine combination using a publicly available COVID-19 chest X-ray dataset. Another interesting work in [86] presents a scheme for detecting coronavirus disease COVID-19 using on-board smartphone sensors. The proposed model enables us to read the signal measurements from smartphone sensors and scan CT images to identify viral pneumonia which can potentially identify coronavirus-related symptoms. It would be useful for radiologists with a smart phone in tracking the development of the disease.

\section{Coronavirus diagnosis and treatment}

In the coronavirus fighting, developing innovative and efficient diagnostic and treatment methods has great significance, deciding the success of fighting COVID-19 disease. AI can come up with solutions to help understand and fight COVID19 via intelligent data analytics [87], [88]. The work in [89] introduces the AI-based model, called COVIDX-Net that includes seven different architectures of deep convolutional neural network models, such as the modified Visual Geometry Group Network (VGG19) and the second version of Google MobileNet, for COVID-19 diagnosis. Each deep neural network model is able to analyze the normalized intensities of the $\mathrm{X}$-ray image to recognize and classify the patient status either negative or positive COVID-19 case. Based on the current situation where there is a lack of public COVID-19 datasets, the learning-based scheme is validated on 50 Chest X-ray images with 25 confirmed positive COVID-19 cases, showing good performances in terms of high accuracy in COVID-19 classification. DL has been also used for the quantification of COVID-19 infection in CT images, as reported in [90]. 
More specifically, a VB-Net neural network is employed to segment COVID-19 infection regions in CT scans, with a training dataset from 249 COVID- 19 patients, and then the model is validated using 300 new COVID-19 patients. The proposed scheme potentially assists radiologists to refine the automatic annotation of each infected case.

AI-based machine learning (ML) has been also utilized to facilitate coronavirus diagnosis and treatment. For instance, a study in [91] introduces an ML model to predict the possible inhibitory synthetic antibodies for the SARS-COV-2 virus. 1933 virus-antibody sequences and their clinical patient neutralization response were collected that have been trained using an ML model to predict the antibody response. The work in [92] considers a based alignment-free approach for an ultrafast, scalable, and accurate classification of whole COVID-19 genomes. Decision tree coupled with supervised learning has been also used for genome analysis from a large dataset of over 5000 unique viral genomic sequences. The authors claim that their scheme can achieve high levels of classification accuracy and discovers the most relevant relationships among over 5,000 viral genomes within a few minutes. Additionally, the research in [93] suggests an ML-based miRNA prediction analysis for the SARS-CoV-2 genome to identify miRNA-like hairpin which can impact the COVID-19 virus. Learning can help to realize potential miRNA based interactions between the viral miRNAs and human genes as well as human miRNAs and viral genes, which are important to identify the mechanisms behind the SARS-CoV-2 infections.

\section{Vaccine/drug development}

In order to ultimately combat the emerging COVID-19 pandemic, it is desired to develop effective vaccines against deadly disease caused by COVID-19 virus. AI can come as an attractive tool to support vaccine production. Some rapidly emerging studies suggest to use AI for this task. For example, the work in [94] employs Vaxign-ML tool to predict 24 COVID-19 vaccine candidates by using the $\mathrm{S}$ protein and five non-structural proteins that aims investigating the entire proteome of CoV-2. By applying reverse vaccinology and machine learning, the authors can predict potential vaccine targets for effective and safe COVID-19 vaccine development. Another vaccine trial is in [95] which leverages HLA-binding prediction tools using an peptide stability assay. 777 peptides were assessed that were predicted to be good binders across 11 MHC allotypes with high prediction binding scores. Preliminary results can make important contributions to the design of an efficacious vaccine against COVID-19.

Up to now, there is no effective treatment for this COVID-19 epidemic. However, the viral protease of a coronavirus can be extracted and replicated for drug discovery. The work in [96] suggests that the potential anti-SARS-CoV chemotherapies can be potential 2019-nCoV drugs. A machine intelligencebased generative network complex model is derived to generate a family of potential 2019-nCoV drugs, inherited from HIV-1 protease [97]. The results from this work can open up new opportunities to seek new drugs for fighting COVID-19 in this crisis.
Unlike the previous work, the study in [98] uses DL to develop a drug-target interaction model called Molecule Transformer-Drug Target Interaction (MT-DTI) to identify commercially available drugs that can act on viral proteins of 2019- nCoV. They suggest that atazanavir, an antiretroviral medication used for HIV fighting, can be useful to develop a chemical compound specific for COVID-19. The efficiency of the discovered drug is under the trial stage before possibly using on corona-infected patients. Moreover, the work in [99] designs a data-driven drug repositioning framework, by integrating ML and statistical analysis approaches to systematically mine large-scale knowledge graph and transcriptome data. Results from trials show that ML can predict effective drug candidates against SARS-CoV-2. Meanwhile, drug repositioning is a possible solution for combating the global coronavirus crisis [100]. A panel of 49 FDA-approved drugs that have been selected by an assay of SARS-CoV is monitored to recognize the potential drug candidates against COVID-19 infection. This trial drugs target to be tested on animals for tracking their antiviral activities before using on human.

\section{E. Prediction of future COVID-19 outbreak}

The recent emergence of coronavirus has highlighted the need for prediction of future outbreak like COVID-19. Epidemic models of COVID to predict and control the outbreak should be taken into consideration in the coronavirus fighting activities [101], [102], [103]. Recently, AI has been applied to predict outbreak like coronavirus. For example, an prediction model using AI to estimate the size, lengths and ending time of Covid-19 across China [104]. An auto-encoder is designed for modelling the transmission dynamics of the epidemic based on dataset collected from WHO sources. Clustering algorithms may be useful to classify different cities, regions for investigating the virus transmissions for outbreak prediction. Another model is developed for outbreak prediction in Mexico, as reported in [105]. The biological features of the virus and the possible pathophysiological mechanisms of its disease and a stochastic model based on a Markov chain are used to express the probability distribution of cases in Mexico states. This also can estimate the number of cases in Mexico that facilitates the government in the estimation of virus spread making appropriate actions for future fightings.

From the statistics collected from the COVID-19 outbreak, a research group in China built a prediction model for future infectious diseases using ML [106]. The key idea is to extend causal inference theory and ML to identify and quantify the most important factors that cause zoonotic disease and COVID-19 outbreaks. Based on that, the model can generate visual tools to illustrate the complex causal relationships of animal infectious diseases and their correlation with COVID19 diseases. Meanwhile, the authors in [107] model the nondeterministic data distributions via DL network and fuzzy rule induction for obtaining better the stochastic insight about the epidemic development. Based on the model outputs, policy makers can have a better picture about the coronavirus spread and estimate the possibility of future similar outbreak. DL has 
been also tested in [108] to build a virus host prediction model for identifying the way the virus transmits, with different sources such as bat coronaviruses, mink viruses and finding the relation with COVID-19. This research potentially discovers the origins of infection and predicts how coronavirus transmits for appropriate preventive actions.

\section{BLOCKCHAIN AND AI USE CASES FOR CORONAVIRUS FIGHTING}

The use of blockchain and AI for solving coronavirus pandemic has been attracted much attention from industry and research community with projects and use case trials. In this section, we highlight the most popular use cases on blockchain and $\mathrm{AI}$ adaption in response to coronavirus fighting.

\section{A. Blockchain use cases}

1) Hashlog: The first project is Hashlog that was created by a Georgia-based health tech startup Acoer [109]. They has come up with a HashLog blockchain solution to combat and control the coronavirus from spreading. This can be enabled by the distributed blockchain ledger technology which ensures logging and data visualization of the coronavirus outbreak from the public data of US Centres for Disease Control (CDC) and WHO. Hashlog potentially provides real-time updates on the transmission of virus by tracking the movement of infected people which allows the healthcare authorities to make decisions for fighting further infections.

2) Hyperchain: The next blockchain project related to coronavirus is Hyperchain [110], which has built a donation tracking platform for supporting governments and healthcare organizations in the donation process to the infected victims in China. Hyperchain can ensure the transparency of the donation records from the origins to the destinations without being changed or modified. Moreover, the Hyperchain is able to connect up to millions of nodes so that more users can reach donated goods and necessary medical equipment from the factories, helping to solve the facility shortage issues during the epidemic.

3) VeChain: One interesting blockchain use case is VeChain which is a blockchain-based platform built for monitoring vaccine production in China [111]. All activities related to vaccine manufacturing from materials, codes to package are recorded and stored on distributed ledgers. This project also provides a reliable method to reduce the risk of potential modifications on vaccine information. VeChain also ensures that vaccine records are immutable and permanent to achieve high vaccine quality which is highly important in the healthcare sector, like COVID-19 epidemic.

4) $P H B C$ : Another promising blockchain platform is PHBC [112] that is a monitoring blockchain for the continual and anonymous verification of communities and workplaces that are free from coronavirus COVID-19 as well as other high-risk viruses, bacteria, to aid them in staying free of deadly diseases. An interesting feature of this blockchain platform is the ability of monitoring the movement of uninfected persons, while restricting their return if they have gone to the areas known to be infected. PHBC can also automatically identify zones with and without validated incident reports by integrating the real-time information collected from virus surveillance providers with AI and geographical information systems.

\section{B. AI use cases}

Various AI companies are also making a play towards efficient detection and diagnostic of diseases caused by COVID19 virus. Some important use cases and projects will be highlighted as follows.

1) Bluedot: Bluedot is an AI project developed by a Toronto-based startup that aims to detect coronavirus in the epidemic [113]. Bluedot use AI algorithms to build prediction models for virus ditection. It even identified the coronavirus outbreak in Wuhan hours before the local authorities had diagnosed the first cases of the virus. Bluedot collects information from social media, government documents, data of healthcare organizations to build intelligence enabled by natural language processing (NLP) and machine learning. It is able to track outbreaks of over 100 different diseases, every 15 minutes around the clock.

2) Infervision: Another emerging AI use case in response to coronavirus pandemic is Infervision [114] that aims to help clinicians detect and monitor the disease efficiently. Infervision has been used at the center of the epidemic outbreak at Tongji Hospital in Wuhan, China. Infervision AI has improved the CT diagnosis speed, which is highly important to accelerate pneumonia diagnosis for early detection of suspected patients. This AI solution has proved very useful in supporting healthcare services in the emergency situations, like coronavirus outbreak.

3) AlphaFold system, Google DeepMind: Very recently, Good DeepMind has introduced an AI tool called AlphaFold [115] which enables the structure predictions of several understudied proteins associated with SARS-CoV-2 virus that causes the coronavirus outbreak. This AI platform is empowered by a DL system and a ML-based free modelling technique to help it estimate protein structures when no similar structures of protein are available. Up to date, AlphaFold is under the trial stages and experiments have yet not been verified.

4) NVIDIA's AI: Last month, a team of physicians at the Zhongnan Hospital, Wuhan China, used GPU-accelerated AI software called NVIDIA's AI, which was primarily used to detect cancer in lung CTs, to identify the visual signs of the COVID-19 virus [116]. Data training was performed by using over 2,000 CT images from some of the first coronavirus patients in China, aiming to build AI models for detecting signs of pneumonia caused by coronavirus. This AI solution assist doctors in the virus recognition for fast diagnosis and treatment.

\section{Challenges AND FUtURE DiRECTIONS}

The application of blockchain and AI technologies is promising to help deal with the coronavirus epidemic. However, the provided throughout survey also reveals several critical challenges and open issues that should be considered carefully when applying these technologies in the context of 
healthcare sector for epidemic. We first highlight the research challenges, then we point out some potential future directions in this field.

\section{A. Challenges}

We here analyse the challenges from four main aspects: regulatory consideration, people's privacy preservation, security of blockchain and AI ecosystem, and the lack of unified databases.

1) Regulatory consideration: The use of blockchain and $\mathrm{AI}$ in the healthcare sector like coronavirus fighting should be considered carefully with regulatory laws. While the features of blockchain and AI can bring benefits, they also poses a legal and regulatory challenge if there is no party that is responsible and can be held accountable. For example, in the blockchain network, it will be important to consider what law might apply to transactions and what appropriate risk management should be put into place [117]. Regarding AI, it might be easier to create some forms of legal schemes and internal governance models that will dictate the governing law for AI operations in healthcare [118]. Specially, we also consider legal issues about content, personal information running on blockchain and AI platforms, such as problems with copyright infringement and defamation.

2) People's privacy preservation: In the coronavirus tracking applications, how to protect peoples privacy is highly important. The governments can use mobile location data to help track the outbreak spread, but this solution must ensure the privacy of user data, especially sensitive information, such as home address, banking details, shopping records, etc. The governmental agencies may impose privacy laws on the user tracking mobile apps to ensure the safety and security to the public [119]. Besides, nowadays many healthcare organizations and institutions are collecting data from their patients via electronic healthcare records that helps monitor the COVID19 disease symptoms and serve treatment [120]. In such healthcare activities, the conflict between data collection and user privacy is inevitable that needs to solved by laws and reinforcement from the related authorities.

3) Security of blockchain and AI ecosystem: Blockchain is widely regarded as secure monitoring platform to ensure safety and privacy healthcare applications, such as COVID19 tracking. However, recent research reports have revealed inherent security weaknesses in blockchain which are related to medical and healthcare systems [23]. Data threats or adversaries can enter the blockchain software to hold the control of the blockchain, which can lead to serious consequences, like modifications of medical transaction or patient data information, raising privacy concerns [121]. Security is also a critical concern of AI systems for healthcare applications in the context of COVID-19 crisis. Data collected from patients, clinical labs and hospitals can be modified by data threats. More importantly, malicious attacks can inject false data or adversarial sample inputs which makes AI learning invalid, while the AI model can be tampered with [122]. Therefore, security issues related to blockchain and AI deployment when solving the healthcare issues in the epidemic should be given high priority during the design and development stages.
4) Lack of unified databases: A critical challenge in the coronavirus fighting is the lack of unified database related coronavirus epidemic such as infected cases, affected areas, and medical supply status. Most of the current coronavirusrelated databases come from individual resources, i.e. social media [123], patient collection [124], [125], but they are not sufficient for large-scale AI operations that potentially creates greater impacts on COVID-19 fighting, compared to the current results. Countries are now reluctant to share database, that make international healthcare organizations like WHO challenging to evaluate the epidemic for fighting effectively the virus spread [126]. In fact, WHO cannot provide appropriate public health guidance without disaggregated data and detailed local epidemic information.

\section{B. Future directions}

We discuss some of the future research directions on blockchain and AI adoption for fighting COVID-19 pandemic.

1) Optimized blockchain for better deployment:

Blockchain platforms should be optimized to achieve better performances in terms of reduced network latency, increased throughput, and improved security which can make blockchain become an ideal choice for emergency healthcare applications like the COVID-19 epidemic. For example, lightweight blockchain design in healthcare are necessary to optimize data verification and transaction communication for ultralowlatency information broadcasting [127]. Another feasible solution is to minimize the size of blockchain by establishing local and private blockchain networks, one of these is responsible to monitor the outbreak in a certain area for fast response. To realize this, building customized ledgers that can placed on local servers in the outbreak area can help to enhance the blockchain performance [128].

2) Improved AI algorithm for better analytic accuracy: The efficiency of intelligent healthcare data analytics, i.e. virus information analysis, mainly depends on the AI algorithms. Developing AI architectures specialized in medical applications might be the key for empowering future intelligent data analytics with the ability to handle multimedia healthcare data. Adaptive AI models should be developed in response to COVID-19-like emergency healthcare, such as AI in predictive modeling, AI in patient monitoring, and AI in emergency department operations [129].

3) Combination with other technologies: To achieve better efficiency in solving epidemic-related issues, blockchain and AI can be incorporated with other technologies to build a comprehensive healthcare system. For instance, recently Taiwan leveraged big data as a promising technology combined with AI to control the virus spread [130]. Alibaba has also integrated AI with cloud computing for supporting coronavirus data analytics [131]. The resourceful storage and high computation capability are the key features that cloud can provide to facilitate AI analytics. More interesting, China has recently exploited the mobility of drones [132] to improve the provisions of medical supplies [133]. In fact, using drones would be the fastest way to deliver essential facilities to the victims in the quarantine areas where all modes of transport 
are interrupted. Besides, drones also help to reinforce the contactless monitoring of the outbreak. In the near future, these promising technologies can be integrated to build highly advanced medical system for coping with the coronavirus-like epidemics.

\section{CONCLUSiONS}

In this paper, we have presented a state-of-art survey on the utilization of blockchain and AI technologies to combat the coronavirus (COVID-19) epidemic. We have first introduced a conceptual architecture which integrates blockchain and AI towards the fighting of coronavirus crisis. Specially, we have extensively discussed the key roles of blockchain for solving the pandemic via five important solutions, including outbreak tracking, user privacy protection, safe day-to-day operations, medical supply chain, and donation tracking. Moreover, the potential of AI for coping with the COVID-19 crisis has been also analysed though five main application domains, namely outbreak estimation, coronavirus detection, coronavirus analytics, vaccine/drug development, and prediction of future coronavirus-like outbreak. Some important use cases and projects using blockchain and AI towards COVID-19 fighting have been also highlighted. Finally, we have pointed out some potential challenges and future directions. We believe our timely survey will shed valuable light on the research of the blockchain and AI for COVID-19 fighting as well as motivate the interested researchers and stateholders to put more efforts into using these promising technologies to combat future coronavirus-like epidemics.

\section{REFERENCES}

[1] P. Zhou, X.-L. Yang, X.-G. Wang, B. Hu, L. Zhang, W. Zhang, H.-R. Si, Y. Zhu, B. Li, C.-L. Huang et al., "A pneumonia outbreak associated with a new coronavirus of probable bat origin," Nature, pp. 1-4, 2020.

[2] C.-C. Lai, T.-P. Shih, W.-C. Ko, H.-J. Tang, and P.-R. Hsueh, "Severe acute respiratory syndrome coronavirus 2 (sars-cov-2) and corona virus disease-2019 (COVID-19): the epidemic and the challenges," International journal of antimicrobial agents, p. 105924, 2020.

[3] Economic Outlook 2020. [Online]. Available: https://www.oecd.org/economic-outlook/.

[4] S. Reddy, J. Fox, and M. P. Purohit, "Artificial intelligence-enabled healthcare delivery," Journal of the Royal Society of Medicine, vol. 112 , no. 1, pp. 22-28, 2019

[5] M. Hölbl, M. Kompara, A. Kamišalić, and L. Nemec Zlatolas, "A systematic review of the use of blockchain in healthcare," Symmetry, vol. 10, no. 10, p. 470, 2018.

[6] A. A. Monrat, O. Schelén, and K. Andersson, "A survey of blockchain from the perspectives of applications, challenges, and opportunities," IEEE Access, vol. 7, pp. 117 134-117 151, 2019.

[7] S. Johnstone, "A viral warning for change. COVID-19 versus the red cross: Better solutions via blockchain and artificial intelligence," COVID-19 Versus the Red Cross: Better Solutions Via Blockchain and Artificial Intelligence (February 3, 2020). University of Hong Kong Faculty of Law Research Paper, no. 2020/005, 2020.

[8] Blockhain, AI and the Wuhan Coronavirus. [Online]. Available: https://www.law.ox.ac.uk/business-law-blog/blog/2020/02/blockhainai-and-wuhan-coronavirus.

[9] Government okays mass surveillance of Israelis phones to curb coronavirus. [Online]. Available: https://www.timesofisrael.com/governmentokays-mass-surveillance-of-israelis-phones-to-curb-coronavirus/.

[10] Coronavirus: Can AI (Artificial Intelligence) Make A Difference? [Online]. Available: https://www.forbes.com/sites/tomtaulli/2020/02/02/coronavirus-can-aiartificial-intelligence-make-a-difference.
[11] Z. Ma, "Spatiotemporal fluctuation scaling law and metapopulation modeling of the novel coronavirus (COVID-19) and sars outbreaks," arXiv preprint arXiv:2003.03714, 2020.

[12] White House, tech industry discuss AI solutions to coronavirus pandemic on 'initial' call. [Online]. Available: https://www.fedscoop.com/coronavirus-white-house-tech-industrycall/.

[13] T. H.-T. W.-J. H. Quoc-Viet Pham, Dinh C. Nguyen and P. N. Pathirana, "Artificial intelligence (AI) and big data for coronavirus (COVID-19) pandemic: A survey on the state-of-the-arts," 10.13140/RG.2.2.23518.38727, 2020

[14] Z. Wu and J. M. McGoogan, "Characteristics of and important lessons from the coronavirus disease 2019 (COVID-19) outbreak in china: summary of a report of 72314 cases from the chinese center for disease control and prevention," Jama, 2020.

[15] J. M. Read, J. R. Bridgen, D. A. Cummings, A. Ho, and C. P. Jewell, "Novel coronavirus 2019-ncov: early estimation of epidemiological parameters and epidemic predictions," MedRxiv, 2020.

[16] Q. Li, X. Guan, P. Wu, X. Wang, L. Zhou, Y. Tong, R. Ren, K. S. Leung, E. H. Lau, J. Y. Wong et al., "Early transmission dynamics in wuhan, china, of novel coronavirus-infected pneumonia," New England Journal of Medicine, 2020.

[17] COVID-19 CORONAVIRUS PANDEMIC. [Online]. Available: https://www.worldometers.info/coronavirus/.

[18] C. Albulescu, "Coronavirus and financial volatility: 40 days of fasting and fear," arXiv preprint arXiv:2003.04005, 2020.

[19] T. Fetzer, L. Hensel, J. Hermle, and C. Roth, "Coronavirus perceptions and economic anxiety," arXiv preprint arXiv:2003.03848, 2020.

[20] X. Wang, X. Zha, W. Ni, R. P. Liu, Y. J. Guo, X. Niu, and K. Zheng, "Survey on blockchain for internet of things," Computer Communications, vol. 136, pp. 10-29, 2019.

[21] D. C. Nguyen, P. N. Pathirana, M. Ding, and A. Seneviratne, "Blockchain for $5 \mathrm{~g}$ and beyond networks: A state of the art survey," arXiv preprint arXiv:1912.05062, 2019.

[22] D. C. Nguyen and et al., "Integration of blockchain and cloud of things: Architecture, applications and challenges," arXiv preprint arXiv:1908.09058, 2019.

[23] C. C. Agbo, Q. H. Mahmoud, and J. M. Eklund, "Blockchain technology in healthcare: a systematic review," in Healthcare, vol. 7, no. 2. Multidisciplinary Digital Publishing Institute, 2019, p. 56.

[24] T.-T. Kuo, H.-E. Kim, and L. Ohno-Machado, "Blockchain distributed ledger technologies for biomedical and health care applications," Journal of the American Medical Informatics Association, vol. 24, no. 6, pp. 1211-1220, 2017.

[25] A. Hasselgren, K. Kralevska, D. Gligoroski, S. A. Pedersen, and A. Faxvaag, "Blockchain in healthcare and health sciences-a scoping review," International Journal of Medical Informatics, p. 104040, 2019.

[26] D. C. Nguyen, P. N. Pathirana, M. Ding, and A. Seneviratne, "Blockchain for secure ehrs sharing of mobile cloud based e-health systems," IEEE access, vol. 7, pp. 66792-66 806, 2019.

[27] D. C. Nguyen, K. D. Nguyen, and P. N. Pathirana, "A mobile cloud based iomt framework for automated health assessment and management," in 2019 41st Annual International Conference of the IEEE Engineering in Medicine and Biology Society (EMBC), 2019, pp. 6517-6520.

[28] S. Jiang, J. Cao, H. Wu, Y. Yang, M. Ma, and J. He, "Blochie: a blockchain-based platform for healthcare information exchange," in 2018 ieee international conference on smart computing (smartcomp), 2018, pp. 49-56.

[29] X. Zheng, R. R. Mukkamala, R. Vatrapu, and J. Ordieres-Mere, "Blockchain-based personal health data sharing system using cloud storage," in 2018 IEEE 20th International Conference on e-Health Networking, Applications and Services (Healthcom), 2018, pp. 1-6.

[30] AI Weekly: Coronavirus, facial recognition, and the future of privacy. [Online]. Available: https://venturebeat.com/2020/03/06/aiweekly-coronavirus-facial-recognition-and-the-future-of-privacy/.

[31] C. M. Bishop, Pattern recognition and machine learning. springer, 2006.

[32] B. J. Erickson, P. Korfiatis, Z. Akkus, and T. L. Kline, "Machine learning for medical imaging," Radiographics, vol. 37, no. 2, pp. 505515, 2017.

[33] S. U. Amin, M. S. Hossain, G. Muhammad, M. Alhussein, and M. A. Rahman, "Cognitive smart healthcare for pathology detection and monitoring," IEEE Access, vol. 7, pp. 10 745-10753, 2019.

[34] Can CCTV help contain the Coronavirus?. [Online]. Available: https://www.ifsecglobal.com/asia/can-cctv-help-contain-coronavirus/. 
[35] S. Pouyanfar, S. Sadiq, Y. Yan, H. Tian, Y. Tao, M. P. Reyes, M.L. Shyu, S.-C. Chen, and S. Iyengar, "A survey on deep learning: Algorithms, techniques, and applications," ACM Computing Surveys (CSUR), vol. 51, no. 5, pp. 1-36, 2018.

[36] A. Shrestha and A. Mahmood, "Review of deep learning algorithms and architectures," IEEE Access, vol. 7, pp. 53 040-53 065, 2019.

[37] G. Litjens, T. Kooi, B. E. Bejnordi, A. A. A. Setio, F. Ciompi, M. Ghafoorian, J. A. Van Der Laak, B. Van Ginneken, and C. I. Sánchez, "A survey on deep learning in medical image analysis," Medical image analysis, vol. 42, pp. 60-88, 2017.

[38] F. Jiang, Y. Jiang, H. Zhi, Y. Dong, H. Li, S. Ma, Y. Wang, Q. Dong, H. Shen, and Y. Wang, "Artificial intelligence in healthcare: past, present and future," Stroke and vascular neurology, vol. 2, no. 4, pp. 230-243, 2017.

[39] R. Miotto, F. Wang, S. Wang, X. Jiang, and J. T. Dudley, "Deep learning for healthcare: review, opportunities and challenges," Briefings in bioinformatics, vol. 19, no. 6, pp. 1236-1246, 2018.

[40] Five Companies Using AI to Fight Coronavirus. [Online]. Available: https://spectrum.ieee.org/the-human-os/artificial-intelligence/medicalai/companies-ai-coronavirus.

[41] S. P. Novikov, O. D. Kazakov, N. A. Kulagina, and N. Y. Azarenko, "Blockchain and smart contracts in a decentralized health infrastructure," in 2018 IEEE International Conference" Quality Management, Transport and Information Security, Information Technologies"(IT\&QM\&IS), 2018, pp. 697-703.

[42] Blockchain in Healthcare: the Case of Coronavirus. [Online]. Available: https://www.e-zigurat.com/innovation-school/blog/blockchain-inhealthcare/.

[43] Blockchain Can Track the Deadly Coronavirus. [Online]. Available: https://www.blockchain-council.org/blockchain/blockchain-can-trackthe-deadly-coronavirus/.

[44] PHBC announces blockchain monitor to track virus-free zones. [Online]. Available: https://www.cryptopolitan.com/phbc-blockchainmonitor-for-virus-free-zones/.

[45] Blockchain Monitor Launched to Track Coronavirus-Free Safe Zones to Protect the Non-Infected Community During Pandemic. [Online]. Available: https://blockchain.news/news/blockchain-monitor-trackcoronavirus-free-zones-protect-non-infected-community-pandemic.

[46] Iran minister accuses 'some countries' of not declaring their coronavirus cases. [Online]. Available: https://www.cnbc.com/2020/03/06/iran-minister-accuses-somecountries-of-not-declaring-coronavirus-cases.html.

[47] How Social Media Is Shaping Our Fears of-and Response to-the Coronavirus . [Online]. Available: https://time.com/5802802/social-mediacoronavirus/.

[48] T. M. Fernández-Caramés and P. Fraga-Lamas, "A review on the use of blockchain for the internet of things," IEEE Access, vol. 6, pp. 32979 $33001,2018$.

[49] Coronavirus Tracking and Surveillance May Have Consequences for the Protection of Personal Data Privacy. [Online]. Available: https://blockchain.news/news/coronavirus-tracking-surveillanceconsequences-protection-personal-data-privacy.

[50] G. Zyskind, O. Nathan et al., "Decentralizing privacy: Using blockchain to protect personal data," in 2015 IEEE Security and Privacy Workshops, 2015, pp. 180-184.

[51] T. Kumar, V. Ramani, I. Ahmad, A. Braeken, E. Harjula, and M. Ylianttila, "Blockchain utilization in healthcare: Key requirements and challenges," in 2018 IEEE 20th International Conference on e-Health Networking, Applications and Services (Healthcom), 2018, pp. 1-7.

[52] D. V. Dimitrov, "Blockchain applications for healthcare data management," Healthcare informatics research, vol. 25, no. 1, pp. 51-56, 2019.

[53] Privacy Advocates Are Sounding Alarms Over Coronavirus Surveillance. [Online]. Available: https://finance.yahoo.com/news/privacyadvocates-sounding-alarms-over-210014068.html.

[54] UAE uses blockchain, digital identity to battle COVID-19. [Online]. Available: https://www.ledgerinsights.com/uae-uses-blockchain-digitalidentity-to-battle-COVID-19/.

[55] Electronic payments look more appealing as people fear cash could spread coronavirus. [Online]. Available: Electronic payments look more appealing as people fear cash could spread coronavirus https://www.cnbc.com/2020/03/16/electronic-payments-look-moreappealing-as-coronavirus-spreads.html.

[56] R. Bhatia, P. Kumar, S. Bansal, and S. Rawat, "Blockchain-the technology of crypto currencies," in 2018 International Conference on Advances in Computing and Communication Engineering (ICACCE), 2018, pp. 372-377.
[57] How blockchain technology is helping businesses overcome the COVID-19 outbreak in China. [Online]. Available: https://irishtechnews.ie/blockchain-businesses-overcome-COVID19-china/.

[58] China taps blockchain technology to boost financing for businesses hit by virus. [Online]. Available: https://www.cnbc.com/2020/02/15/coronavirus-china-taps-blockchaintech-to-help-firns-hit-by-virus.html.

[59] N. Kshetri and J. Voas, "Blockchain in developing countries," It Professional, vol. 20, no. 2, pp. 11-14, 2018.

[60] Blockchain Against Hunger: Harnessing Technology In Support Of Syrian Refugees. [Online]. Available: https://www.wfp.org/news/blockchain-against-hunger-harnessingtechnology-support-syrian-refugees.

[61] H. Juma, K. Shaalan, and I. Kamel, "A survey on using blockchain in trade supply chain solutions," IEEE Access, 2019.

[62] P. Gonczol, P. Katsikouli, L. Herskind, and N. Dragoni, "Blockchain implementations and use cases for supply chains-a survey," Ieee Access, vol. 8, pp. 11 856-11 871, 2020.

[63] M. Kouhizadeh and J. Sarkis, "Blockchain practices, potentials, and perspectives in greening supply chains," Sustainability, vol. 10, no. 10, p. 3652, 2018.

[64] H. Wu, J. Cao, Y. Yang, C. L. Tung, S. Jiang, B. Tang, Y. Liu, X. Wang, and Y. Deng, "Data management in supply chain using blockchain: Challenges and a case study," in 2019 28th International Conference on Computer Communication and Networks (ICCCN), 2019, pp. 1-8.

[65] Alipay Launches Blockchain Based Platform To Help Combat CoronaVirus. [Online]. Available: https://coingape.com/alipaylaunches-blockchain-based-platform-to-help-combat-corona-virus-aschina-shuts-down-mining-farms/.

[66] Five Ways Blockchain Can Unblock The Coronavirus Medical Supply Chain. [Online]. Available: forbes.com/sites/nishandegnarain/2020/03/22/5-ways-blockchaincan-unblock-the-coronavirus-medical-supply-chain/.

[67] N. S. Sirisha, T. Agarwal, R. Monde, R. Yadav, and R. Hande, "Proposed solution for trackable donations using blockchain," in 2019 International Conference on Nascent Technologies in Engineering (ICNTE), 2019, pp. 1-5.

[68] H. Saleh, S. Avdoshin, and A. Dzhonov, "Platform for tracking donations of charitable foundations based on blockchain technology," in 2019 Actual Problems of Systems and Software Engineering (APSSE), 2019, pp. 182-187.

[69] Blockchain and AI Amidst the Coronavirus Crisis: 'A Call to Arms' . [Online]. Available: https://www.cryptonewsz.com/blockchain-and-aiamidst-the-coronavirus-crisis-a-call-to-arms/.

[70] Fury in China as footage appears to show officials taking doctors' face masks. [Online]. Available: https://www.theguardian.com/world/2020/feb/02/shameless-outragechina-coronavirus-outbreak-mask.

[71] Italian Red Cross Coronavirus Bitcoin Fundraiser Smashes Goal, Issues New Initiative. [Online]. Available: https://cointelegraph.com/news/italian-red-cross-coronavirus-bitcoinfundraiser-smashes-goal-issues-new-initiative.

[72] Coronavirus Relief: Cryptocurrency Aid Programs Launched to Combat COVID-19 Outbreak. [Online]. Available: https://news.bitcoin.com/coronavirus-relief-aid-cryptocurrency/.

[73] The role of wireless tech in fighting the new coronavirus. [Online]. Available: https://www.lightreading.com/4g-3g-wifi/the-role-ofwireless-tech-in-fighting-the-new-coronavirus/d/d-id/757634.

[74] I. H. Sarker, "Context-aware rule learning from smartphone data: survey, challenges and future directions," Journal of Big Data, vol. 6 , no. 1, p. 95, 2019.

[75] I. H. Sarker, A. Kayes, and P. Watters, "Effectiveness analysis of machine learning classification models for predicting personalized context-aware smartphone usage," Journal of Big Data, vol. 6, no. 1, p. $57,2019$.

[76] J. Shen and M. O. Shafiq, "Learning mobile application usage-a deep learning approach," in 2019 18th IEEE International Conference On Machine Learning And Applications (ICMLA), 2019, pp. 287-292.

[77] U.S. uses location data to track movements during coronavirus: report. [Online]. Available: https://www.fiercewireless.com/wireless/ususes-location-data-to-track-movements-during-coronavirus.

[78] Use of Twitter social media activity as a proxy for human mobility to predict the spatiotemporal spread of 2019 novel coronavirus at global level. [Online]. Available: https://www.rti.org/publication/use-twittersocial-media-activity-proxy-human-mobility-predict-spatiotemporalspread. 
[79] Z. Wang, G. Wang, B. Huang, Z. Xiong, Q. Hong, H. Wu, P. Yi, K. Jiang, N. Wang, Y. Pei et al., "Masked face recognition dataset and application," arXiv preprint arXiv:2003.09093, 2020.

[80] Y. Wang, M. Hu, Q. Li, X.-P. Zhang, G. Zhai, and N. Yao, "Abnormal respiratory patterns classifier may contribute to large-scale screening of people infected with COVID-19 in an accurate and unobtrusive manner," arXiv preprint arXiv:2002.05534, 2020.

[81] O. Gozes, M. Frid-Adar, H. Greenspan, P. D. Browning, H. Zhang, W. Ji, A. Bernheim, and E. Siegel, "Rapid ai development cycle for the coronavirus (COVID-19) pandemic: Initial results for automated detection \& patient monitoring using deep learning ct image analysis," arXiv preprint arXiv:2003.05037, 2020.

[82] X. Xu, X. Jiang, C. Ma, P. Du, X. Li, S. Lv, L. Yu, Y. Chen, J. Su, G. Lang et al., "Deep learning system to screen coronavirus disease 2019 pneumonia," arXiv preprint arXiv:2002.09334, 2020.

[83] A. Narin, C. Kaya, and Z. Pamuk, "Automatic detection of coronavirus disease (COVID-19) using X-ray images and deep convolutional neural networks," arXiv preprint arXiv:2003.10849, 2020.

[84] A. Lopez-Rincon, A. Tonda, L. Mendoza-Maldonado, E. Claassen, J. Garssen, and A. D. Kraneveld, "Accurate identification of sars-cov-2 from viral genome sequences using deep learning," bioRxiv, 2020.

[85] B. Ghoshal and A. Tucker, "Estimating uncertainty and interpretability in deep learning for coronavirus (COVID-19) detection," arXiv preprint arXiv:2003.10769, 2020.

[86] H. S. Maghdid, K. Z. Ghafoor, A. S. Sadiq, K. Curran, and K. Rabie, "A novel ai-enabled framework to diagnose coronavirus covid 19 using smartphone embedded sensors: Design study," arXiv preprint arXiv:2003.07434, 2020

[87] M. L. Chiusano, "The modelling of covid19 pathways sheds light on mechanisms, opportunities and on controversial interpretations of medical treatments. v2," arXiv preprint arXiv:2003.11614, 2020.

[88] M. R. Basiri, "Theory about treatments and morbidity prevention of corona virus disease (COVID-19)," Journal of Pharmacy and Pharmacology, vol. 8, pp. 89-90, 2020.

[89] E. E.-D. Hemdan, M. A. Shouman, and M. E. Karar, "Covidx-net: A framework of deep learning classifiers to diagnose COVID-19 in x-ray images," arXiv preprint arXiv:2003.11055, 2020.

[90] F. Shan+, Y. Gao+, J. Wang, W. Shi, N. Shi, M. Han, Z. Xue, D. Shen, and Y. Shi, "Lung infection quantification of COVID-19 in ct images with deep learning," arXiv preprint arXiv:2003.04655, 2020.

[91] R. Magar, P. Yadav, and A. B. Farimani, "Potential neutralizing antibodies discovered for novel corona virus using machine learning," arXiv preprint arXiv:2003.08447, 2020.

[92] G. S. Randhawa, M. P. Soltysiak, H. El Roz, C. P. de Souza, K. A. Hill, and L. Kari, "Machine learning using intrinsic genomic signatures for rapid classification of novel pathogens: COVID-19 case study," bioRxiv, 2020

[93] M. D. S. Demirci and A. Adan, "Computational analysis of micrornamediated interactions in sars-cov-2 infection," bioRxiv, 2020

[94] E. Ong, M. U. Wong, A. Huffman, and Y. He, "COVID-19 coronavirus vaccine design using reverse vaccinology and machine learning," BioRxiv, 2020.

[95] M. Prachar, S. Justesen, D. B. Steen-Jensen, S. P. Thorgrimsen, E. Jurgons, O. Winther, and F. O. Bagger, "COVID-19 vaccine candidates: Prediction and validation of 174 sars-cov-2 epitopes," bioRxiv, 2020.

[96] D. D. Nguyen, K. Gao, R. Wang, and G. Wei, "Machine intelligence design of 2019-ncov drugs," bioRxiv, 2020.

[97] M. R. Dayer, "Old drugs for newly emerging viral disease, COVID-19: Bioinformatic prospective," arXiv preprint arXiv:2003.04524, 2020.

[98] B. R. Beck, B. Shin, Y. Choi, S. Park, and K. Kang, "Predicting commercially available antiviral drugs that may act on the novel coronavirus (2019-ncov), wuhan, china through a drug-target interaction deep learning model," bioRxiv, 2020.

[99] Y. Ge, T. Tian, S. Huang, F. Wan, J. Li, S. Li, H. Yang, L. Hong, $\mathrm{N}$. Wu, E. Yuan et al., "A data-driven drug repositioning framework discovered a potential therapeutic agent targeting COVID-19," bioRxiv, 2020.

[100] S. Jeon, M. Ko, J. Lee, I. Choi, S. Y. Byun, S. Park, D. Shum, and S. Kim, "Identification of antiviral drug candidates against sars-cov-2 from fda-approved drugs," bioRxiv, 2020.

[101] S. S. Nadim, I. Ghosh, and J. Chattopadhyay, "Short-term predictions and prevention strategies for covid-2019: A model based study," arXiv preprint arXiv:2003.08150, 2020.

[102] G. Vattay, "Predicting the ultimate outcome of the COVID-19 outbreak in italy," arXiv preprint arXiv:2003.07912, 2020.
[103] D. Fanelli and F. Piazza, "Analysis and forecast of COVID-19 spreading in china, italy and france," Chaos, Solitons \& Fractals, vol. 134, p. 109761, 2020.

[104] Z. Hu, Q. Ge, L. Jin, and M. Xiong, "Artificial intelligence forecasting of COVID-19 in china," arXiv preprint arXiv:2002.07112, 2020

[105] A. Vivanco-Lira, "Predicting COVID-19 distribution in mexico through a discrete and time-dependent markov chain and an sir-like model," arXiv preprint arXiv:2003.06758, 2020.

[106] L. Jia, K. Li, Y. Jiang, X. Guo et al., "Prediction and analysis of coronavirus disease 2019," arXiv preprint arXiv:2003.05447, 2020.

[107] S. J. Fong, G. Li, N. Dey, R. G. Crespo, and E. Herrera-Viedma, "Composite monte carlo decision making under high uncertainty of novel coronavirus epidemic using hybridized deep learning and fuzzy rule induction," arXiv preprint arXiv:2003.09868, 2020.

[108] H. Zhu, Q. Guo, M. Li, C. Wang, Z. Fang, P. Wang, J. Tan, S. Wu and Y. Xiao, "Host and infectivity prediction of wuhan 2019 novel coronavirus using deep learning algorithm," bioRxiv, 2020

[109] Acoer Coronavirus Tracker, Powered by Hedera Hashgraph, Now Freely Available to General Public with Added Clinical Trial Data. [Online]. Available: https://www.hedera.com/blog/acoer-coronavirustracker-powered-by-hedera-hashgraph-now-freely-available-to-generalpublic-with-added-clinical-trial-data.

[110] HyperChain-Blockchain Charity Platform to Fight Against the Coronavirus. [Online]. Available: https://www.hyperchain.cn/en.

[111] VeChain Announces Blockchain Vaccine Tracing Solution for China. [Online]. Available: https://www.nasdaq.com/articles/vechainannounces-blockchain-vaccine-tracing-solution-china-2018-08-16.

[112] Public Health Blockchain Consortium: PHBC . [Online]. Available: https://www.phbconsortium.org/.

[113] BlueDot: Outbreak Risk Software. [Online]. Available: https://bluedot.global/.

[114] Infervision in the Frontlines Against the Coronavirus. [Online]. Available: https://www.infervision.com/.

[115] Computational predictions of protein structures associated with COVID-19. [Online]. Available: https://deepmind.com/research/opensource/computational-predictions-of-protein-structures-associatedwith-COVID-19.

[116] AI Helps Doctors Diagnose the Coronavirus. [Online]. Available: https://news.developer.nvidia.com/ai-helps-doctors-diagnose-thecoronavirus/.

[117] N. Fabiano, "Internet of things and blockchain: legal issues and privacy. the challenge for a privacy standard," in 2017 IEEE International Conference on Internet of Things (iThings) and IEEE Green Computing and Communications (GreenCom) and IEEE Cyber, Physical and Social Computing (CPSCom) and IEEE Smart Data (SmartData), 2017, pp. 727-734.

[118] S. Reddy, "Use of artificial intelligence in healthcare delivery," in eHealth-Making Health Care Smarter. IntechOpen, 2018.

[119] Coronavirus-tracking smartphone apps don't invade privacy says data watchdog. [Online]. Available: https://www.zdnet.com/article/coronavirus-tracking-mobile-appsget-green-light-from-data-watchdog/.

[120] Coronavirus accelerates China's big data collection but privacy concerns remain. [Online]. Available: https://www.scmp.com/tech/appssocial/article/3052232/coronavirus-accelerates-chinas-big-datacollection-privacy.

[121] X. Li, P. Jiang, T. Chen, X. Luo, and Q. Wen, "A survey on the security of blockchain systems," Future Generation Computer Systems, 2017.

[122] Q. Liu, T. Liu, Z. Liu, Y. Wang, Y. Jin, and W. Wen, "Security analysis and enhancement of model compressed deep learning systems under adversarial attacks," in 2018 23rd Asia and South Pacific Design Automation Conference (ASP-DAC), 2018, pp. 721-726.

[123] E. Chen, K. Lerman, and E. Ferrara, "COVID-19: The first public coronavirus twitter dataset," arXiv preprint arXiv:2003.07372, 2020.

[124] J. P. Cohen, P. Morrison, and L. Dao, "COVID-19 image data collection," arXiv preprint arXiv:2003.11597, 2020.

[125] W. Liu, P. T.-W. Yen, and S. A. Cheong, "Coronavirus disease 2019 (COVID-19) outbreak in china, spatial temporal dataset," arXiv preprint arXiv:2003.11716, 2020

[126] The World Has a Plan to Fight Coronavirus. [Online]. Available: https://www.nytimes.com/2020/03/12/world/coronavirus-world-healthorganization.html.

[127] Y. Liu, K. Wang, Y. Lin, and W. Xu, "Lightchain: A lightweight blockchain system for industrial internet of things," IEEE Transactions on Industrial Informatics, vol. 15, no. 6, pp. 3571-3581, 2019. 
[128] W. J. Gordon and C. Catalini, "Blockchain technology for healthcare: facilitating the transition to patient-driven interoperability," Computational and structural biotechnology journal, vol. 16, pp. 224-230, 2018.

[129] N. Liu, Z. Zhang, A. W. Ho, and M. E. H. Ong, "Artificial intelligence in emergency medicine," Journal of Emergency and Critical Care Medicine, vol. 2, p. 82, 2018.

[130] Big Data Helps Taiwan Fight Coronavirus. [Online]. Available: https://spectrum.ieee.org/the-human-os/biomedical/devices/big-datahelps-taiwan-fight-coronavirus.

[131] Alibaba Cloud offers AI platform to support coronavirus medical efforts. [Online]. Available: https://www.zdnet.com/article/alibaba-cloudoffers-ai-platform-to-support-coronavirus-medical-research/.

[132] D. Chi-Nguyen, P. N. Pathirana, M. Ding, and A. Seneviratne, "Secrecy performance of the uav enabled cognitive relay network," in 2018 IEEE 3rd International Conference on Communication and Information Systems (ICCIS), 2018, pp. 117-121.

[133] China fights coronavirus with delivery drones. [Online]. Available: https://www.gpsworld.com/china-fights-coronavirus-with-deliverydrones/. 